\title{
The effect of multi-vitamin/mineral supplementation on mortality during treatment of pulmonary tuberculosis: a randomised two-by-two factorial trial in Mwanza, Tanzania
}

\author{
Nyagosya Range ${ }^{1}$, John Changalucha ${ }^{2}$, Henrik Krarup ${ }^{3}$, Pascal Magnussen ${ }^{4}$, Åse B. Andersen ${ }^{5}$ and \\ Henrik Friis ${ }^{6} *$ \\ ${ }^{1}$ National Institute for Medical Research, Muhimbili Research Station, PO Box 3436, Dar es Salaam, Tanzania \\ ${ }^{2}$ National Institute for Medical Research, Mwanza Medical Research Centre, PO Box 1462, Mwanza, Tanzania \\ ${ }^{3}$ Department of Clinical Biochemistry, Aalborg University Hospital, PO Box 561, 9100 Aalborg, Denmark \\ ${ }^{4}$ DBL Institute for Health Research and Development, Jaegersborg Allé 1D, Charlottenlund, Denmark \\ ${ }^{5}$ Department of Infectious Diseases, Rigshospitalet, University of Copenhagen, Blegdamsvej 9, 2100 Copenhagen $\emptyset$, Denmark \\ ${ }^{6}$ Department of Epidemiology, Institute of Public Health, University of Copenhagen, Øster Farimagsgade 5B, DK-1014 \\ Copenhagen K, Denmark
}

(Received 2 August 2005 - Revised 11 November 2005 - Accepted 22 November 2005)

\begin{abstract}
Malnutrition is common in pulmonary tuberculosis (TB), and may impair survival. The objective of this study was to assess effects of multi-vita$\mathrm{min} /$ mineral (MVM) and zinc (Zn) supplementation during TB treatment on mortality. Patients diagnosed with sputum-positive pulmonary TB in Mwanza, Tanzania, were randomised, using a two-by-two factorial design, to Zn (45 mg) or placebo, and MVM (vitamins A, B, C, D, E, and selenium and copper) or placebo. Survival status was ascertained at the end of the 8-month TB treatment and supplementation period. Of 499 TB patients, 213 (43\%) had HIV. The mean weight gain at 7 months was $6.88 \mathrm{~kg}$ (95\% CI 6.36, 7.41). Zn and MVM combined, but neither alone (interaction, $P=0.03)$, increased weight gain by $2.37 \mathrm{~kg}(95 \%$ CI $0.91,3.83)$, irrespective of HIV status. Survival status at 8 months was determined for 422 patients $(84.6 \%)$, of which fifty-two (12.3\%) had died. Among fifty-two deaths, there were no effects of MVM (relative risk (RR) $0.73 ; 95 \%$ CI 0.43, 1.23) and Zn (RR 0.76; $95 \%$ CI 0.46, 1.28). However, among HIV co-infected patients, marginally significant effects of both MVM (RR 0.60; $95 \%$ CI 0.34, 1.05) and Zn (RR 0.63, $95 \%$ CI 0.37, 1.08) were seen, and MVM and Zn combined reduced mortality (RR $0 \cdot 29 ; 95 \%$ CI 0.10, 0.80; interaction ratio 0.52). In conclusion, supplementation with MVM, including Zn, during treatment of pulmonary TB may reduce mortality in those co-infected with HIV. A randomised trial of the effect of the combined intervention used in this study should be conducted in a different setting to confirm the finding.
\end{abstract}

Tuberculosis: HIV: Vitamins: Minerals: Weight: Mortality: Tanzania

The risk of active tuberculosis (TB) disease depends on exposure to Mycobacterium tuberculosis and reduced host defence. One-third of the world's population is infected, nine million develop clinical disease each year, and two million die due to the infection (Frieden et al. 2003). HIV infection is considered the strongest single risk factor of developing TB disease after primary infection, and of death during treatment.

Deficiencies of vitamins and minerals, leading to nutritionally acquired immune deficiency syndrome (Beisel, 2001) may be another important determinant of TB infection and disease, as well as poor treatment outcomes (Cegielski \& McMurray, 2004). For example, it is well-documented that zinc ( $\mathrm{Zn}$ ) supplementation reduces risk and severity of diarrhoea and pneumonia in children (Bhutta et al. 1999, 2000), and it has been shown that micronutrient supplementation reduces progression of HIV to AIDS and death in adults (Fawzi et al. 2004; Jiamton et al. 2003).

Despite appropriate anti-TB chemotherapy, the usual cereal-based diet will not ensure an adequate micronutrient intake during convalescence. Micronutrient deficiencies not only impair host immune functions, but may also affect efficacy of TB drugs (Thurnham, 2005), and impair weight gain which may be important to survival (Paton et al. 2004). Although nutritional supplementation during the short-course TB treatment seems logistically feasible and affordable, and may improve treatment outcomes, nutritional assessment and support is currently not part of the case-management.

We conducted a randomised, placebo-controlled, doubleblind, two-by-two factorial trial to assess the effect of daily $\mathrm{Zn}$ and multi-vitamin/mineral (MVM) supplementation on treatment outcomes. Although we found no beneficial effect 
on sputum conversion (Range et al. 2005), the effects on weight gain and mortality over the full duration of TB treatment were also assessed and are reported here.

\section{Subjects and methods}

New or relapse sputum microscopy-positive TB patients aged 15 years or above and resident in the area were consecutively recruited from five health facilities in Mwanza and Magu districts, Mwanza Region, Tanzania, between August 2001 and July 2002. Patients were given information about the study and offered inclusion after informed consent. TB patients returning to treatment after defaulting or smear-positive failure cases, or those with serious TB or other disease unlikely to survive, and pregnant or lactating women were excluded. The study was conducted within the framework of the National TB and Leprosy Programme (Ministry of Health, 1991), with diagnosis, classification, registration and treatment of $\mathrm{TB}$ patients done in accordance with recommended standard procedures (World Health Organization, 2003). In brief, new TB cases were given a combination of four drugs daily under supervision of a health worker during the initial 2-month intensive phase, while two drugs were self-administered daily by the patient, during the 6-month continuation phase.

TB suspects attending in- and out-patient clinics at any of the recruitment centres were asked to submit three sputum specimens (spot, early morning, spot) for examination of acid-fast bacilli using the Ziehl-Neelsen staining technique. Those found sputum microscopy-positive were requested to submit a morning sputum specimen in a sterile universal bottle for confirmation at the Zonal TB Reference Laboratory at Bugando Medical Centre, based on microscopy after fluorochrome (Auramine O) staining, and culture on Lowenstein Jensen solid media (Githui et al. 1993). Only those confirmed to be sputum microscopy- or culture-positive at the Zonal TB Reference Laboratory were included in the trial (Fig. 1).

All patients found sputum microscopy-positive at the recruitment centres were started on $\mathrm{TB}$ treatment, and given a study number and supplement according to the randomisation, as described later. Prior to that, the TB Clinical Officers collected demographic, socio-economic and medical history data from all patients, examined the patients anthropometrically, and collected stool, urine and blood samples for later diagnosis of parasitic infections (World Health Organization, 1991), and determination of $\mathrm{Hb}$ concentration and leucocyte counts. Heights were measured to the nearest $0.1 \mathrm{~cm}$ and weights to the nearest $0.1 \mathrm{~kg}$, with the patient barefoot and wearing light clothing. The same staff member measured the patient at baseline and follow-up. Patients found anaemic or infected with Schistosoma spp., intestinal helminths or malaria were treated accordingly (World Health Organization, 1995). HIV testing was done using two different ELISA. In samples found positive, HIV-1 load was determined using a modification of an in-house developed RT-PCR (Krarup et al. 1998). CD4 cell count was determined by manual immunocytochemistry (Gomo et al. 2004).

\section{Study design and intervention}

The study was a randomised, placebo-controlled trial, using a two-by-two factorial design, to assess the effects of daily supplementation with a MVM and a Zn tablet. Although sputum conversion and weight gain during the 2-month intensive phase were the main outcomes on which sample size considerations were based as previously reported (Range et al. 2005), daily Zn and MVM supplementation was continued throughout the 8 months of short-course TB treatment when survival status was assessed. The MVM tablets contained vitamin A $(1.5 \mathrm{mg})$, vitamin $B_{1}(20 \mathrm{mg})$, vitamin $B_{2}(20 \mathrm{mg})$, vitamin $\mathrm{B}_{6}(25 \mathrm{mg})$, vitamin $\mathrm{B}_{12}(50 \mu \mathrm{g})$, folic acid $(0.8 \mathrm{mg})$, niacin $(40 \mathrm{mg})$, vitamin $\mathrm{C}(200 \mathrm{mg})$, vitamin $\mathrm{E}(60 \mathrm{mg})$, vitamin $\mathrm{D}_{3}$ $(5 \mu \mathrm{g})$, selenium $(0 \cdot 2 \mathrm{mg})$ and copper $(5 \mathrm{mg})$, and $\mathrm{Zn}$ tablets contained $45 \mathrm{mg}$ elementary $\mathrm{Zn}$ (Table 1 ). The rationale for our interventions was the high requirements during TB treatment, when considerable lean body mass is being synthesised. As for the vitamins, the doses were based on the multivitamin supplement proven beneficial among HIV-infected women (Fawzi et al. 1999). However, we included more vitamin $\mathrm{E}$ (60 instead of $30 \mathrm{mg}$ ), since higher doses have been shown to improve immune functions (Food and Nutrition Board, 2000, pp. 186-283), and less vitamin C (200 instead of $1000 \mathrm{mg}$ ), since there has been concern that high doses may cause oxidative damage in individuals with high iron stores (Food and Nutrition Board, 2000, pp. 95-185). However, we included preformed vitamin A only, since it has been

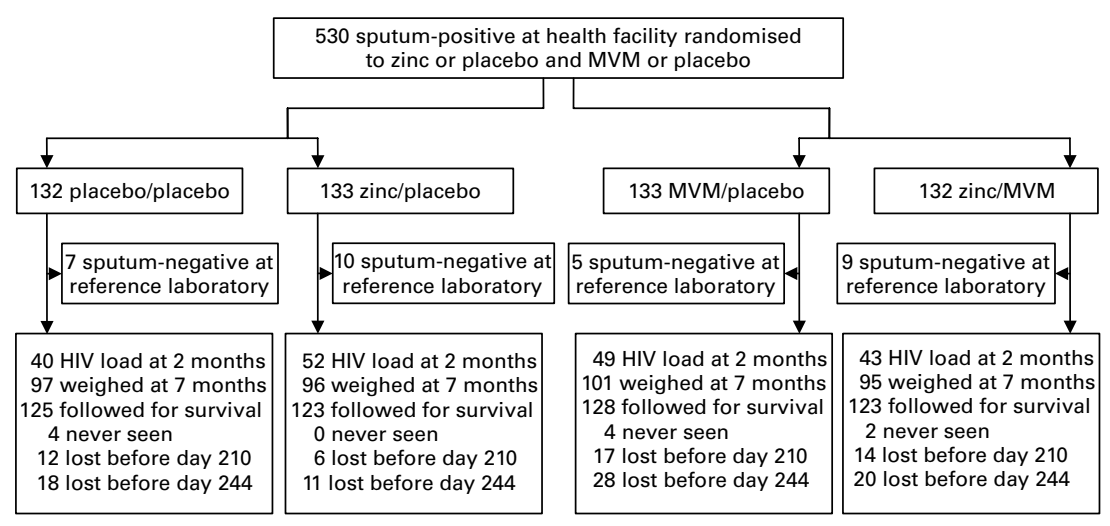

Fig. 1. Trial profile. Sputum-positive pulmonary tuberculosis patients were randomised to daily supplementation with zinc or placebo and, independently, to multivitamin/mineral (MVM) or placebo. Those found sputum-negative at the reference laboratory after randomisation were excluded. 
Table 1. Composition and doses of vitamins and minerals in the two interventions

\begin{tabular}{ll}
\hline & Dose \\
\hline Multi-vitamin/mineral & \\
Vitamin A & $1.5 \mathrm{mg}$ \\
Vitamin $\mathrm{B}_{1}$ & $20 \mathrm{mg}$ \\
Vitamin $\mathrm{B}_{2}$ & $20 \mathrm{mg}$ \\
Vitamin $\mathrm{B}_{6}$ & $25 \mathrm{mg}$ \\
Vitamin $\mathrm{B}_{12}$ & $50 \mu \mathrm{g}$ \\
Folic acid & $0.8 \mathrm{mg}$ \\
Niacine & $40 \mathrm{mg}$ \\
Vitamin C & $200 \mathrm{mg}$ \\
Vitamin $\mathrm{D}_{3}$ & $5 \mu \mathrm{g}$ \\
Vitamin E & $60 \mathrm{mg}$ \\
Selenium (as methionine) & $0.2 \mathrm{mg}$ \\
Copper (as sulphate) & $5 \mathrm{mg}$ \\
Zinc & \\
Zinc (as gluconate) & $45 \mathrm{mg}$
\end{tabular}

postulated that previously observed adverse effects of vitamin A interventions could be due to provitamin carotenoids (Friis, 2005). We also added vitamin D, since vitamin D deficiency has been suggested as a risk factor of TB (Wilkinson et al. 2000). The minerals selenium and copper were added, due to their importance to the immune system (Beisel, 2001). Due to the importance of $\mathrm{Zn}$ to immune functions, and to susceptibility to respiratory and other infections, and to synthesis of lean body mass (Friis \& Sandström, 2001), we assessed its effect separately, using a two-by-two factorial design. The dose of $\mathrm{Zn}$ (45 mg) was considered appropriate, given the low bioavailability of $\mathrm{Zn}$ in populations subsisting on a cereal-based diet (Friis \& Sandström, 2001). Placebo tablets were identical in colour, shape and size to the corresponding white $\mathrm{Zn}$ and green MVM tablets. All tablets were manufactured by Almega (Ringsted, Denmark). Tablets were packed in identical white plastic containers and delivered labelled with four different alphabetical letters. Containers with white tablets ( $\mathrm{Zn}$ and placebo) were labelled either $\mathrm{T}$ or $\mathrm{H}$, and containers with green tablets (MVM and placebo) were labelled either X or S. Before recruitment of patients, all the letters on the containers were replaced with study serial numbers from 1 to 550 based on the computer-generated random sequences, using permuted blocks of four. The five recruitment centres assigned consecutive identity numbers, using different starting numbers, to patients being recruited. Each participant was assigned two containers labelled with the corresponding identity number: one containing white ( $\mathrm{Zn}$ or placebo) and the other green (MVM or placebo) tablets enough for the full 8-month supplementation. Containers were kept in the health facilities where the participants were attending TB treatment. The codes for the MVM and $\mathrm{Zn}$ tablets remained in a sealed envelope, and were only broken after completion of the initial data analysis.

The same health personnel administering TB drugs during the intensive phase ensured that the participant also received the trial tablets under direct observation. At the monthly visits during the continuation phase, each participant was given two plastic bags, appropriately labelled with the patient's identity number, containing thirty white ( $\mathrm{Zn}$ or placebo) and thirty green (MVM or placebo) tablets to take at home along with TB drugs.
The profile for recruitment, randomisation and trial participants is shown in Fig. 1. Trial participants were asked to come to the recruitment centre after 2, 5 and 7 months. Collection of blood was repeated at the 2-month visit, and anthropometric assessment and collection of sputum were repeated at all follow-up visits. Those not seen at the clinic during scheduled follow-up visits were followed up by the study team using addresses given during registration. In cases where the patient had died, the date of death was obtained from relatives. In the analysis of the effect of supplementation on mortality, a person was considered to have died if death occurred within the 8-month supplementation, i.e. not later than $244 \mathrm{~d}$ after start of treatment and supplementation, and to have survived if examined at the 7-month visit or known to be alive later. Cause of death was not ascertained.

The sample size of 500 was initially based on sputum culture conversion and weight gain as the primary outcomes, as previously reported (Range et al. 2005). However, assuming a $10 \%$ cumulative mortality during the 8 months of treatment we would be able to detect a reduction to $5 \%$ or less with $80 \%$ power and $95 \%$ confidence, while allowing for $10 \%$ loss to follow-up.

Permission to conduct the study was granted by the Ethics Committee of the National Institute for Medical Research in Tanzania, and the Danish Central Medical Ethics Committee also approved the study. Permission was also obtained from the Regional Medical Officer and the Council Medical Officers in Mwanza Region. Informed oral consent was obtained from all study participants. Pre-test HIV counselling was given to all, and post-test counselling was given to those who wanted to know their HIV results. Antiretroviral treatment was not available.

\section{Statistical analysis}

Two-sample $t$ test and one-way ANOVA were used to test for differences in means, and $\chi^{2}$-test to test for differences in proportions between groups. Assessment of the effect of the study interventions on mortality was done prior to breaking the code. The analysis was based on intention-to-treat, and done with and without stratification for HIV status. Multiple logistic regression analysis was used to estimate the effects of the study interventions while controlling for potential baseline differences, and to assess for interactions. Binreg was used to compute relative risks (RR), rather than odds ratios, using Stata version 8.2 (StataCorp LP, College Station TX, USA). Survival analysis using Cox regressions was also done, with follow-up time to either the date of death, the date last to follow-up or end of supplementation period. $P$ values below 0.05 were considered significant.

\section{Results}

Of 530 patients found positive based on detection of tubercle bacilli in sputum on microscopy and randomised to the two study interventions, thirty-one could not be confirmed positive based on re-examination at the reference laboratory and were excluded from the study (Fig. 1). Thus, 499 confirmed sputum-positive cases were included in the trial. The mean age was 35.4 (range 15-85) years, and $41.1 \%$ were women. Most of the participants were recruited at Sekou Toure 
Hospital (47.7\%), and the rest from Bugando Medical Centre (16.6\%), Magu Hospital (18.0\%), and Buzuruga (10.2\%) and Butimba (7.4\%) Health Centres. The prevalence of HIV infection was $42.7 \%$ (213), and $34.7 \%$ (173) were found to excrete Schistosoma mansoni and $20.3 \%$ (101) hookworm eggs, and $4.6 \%$ had malaria parasitaemia. As seen in Table 2, baseline equivalence was achieved with respect to age, $\mathrm{BMI}, \mathrm{Hb}$, and prevalence of heavy culture intensity and HIV, although there seemed to be more women in the placobo/placebo group.

As seen from the trial profile (Fig. 1), viral load and CD4 counts were determined after 2 months, weight at 7 months, and survival up to the end of treatment and supplementation at 8 months.

\section{Viral load and CD4 count}

At the 2-month follow-up examination, viral load and CD4 count data were determined in $184(86.4 \%)$ of the 213 HIVinfected participants (Table 3). The mean viral load increase was $0.23 \log _{10}(\mathrm{geq} / \mathrm{l})(95 \% \mathrm{CI} 0.01,0 \cdot 46)$. There was no significant effect of MVM $\left(-0 \cdot 22 \log _{10}(\mathrm{geq} / \mathrm{l}) ; 95 \% \mathrm{CI}-0 \cdot 67\right.$, $0.23 ; \quad P=0.35)$ or $\mathrm{Zn}$ supplementation $\left(0.27 \log _{10}(\mathrm{geq} / \mathrm{l})\right.$, $95 \% \mathrm{CI}-0.18,0.72 ; P=0.24$ ) (interaction, $P=0.37$ ), and no effect of any combination of the two interventions. Zn supplementation alone was associated with a $0.54 \log _{10}(\mathrm{geq} / \mathrm{l})$ (95\% CI $-0.08,1.15 ; P=0.08)$ higher increase. Similarly, there were neither effects of $\mathrm{Zn}(18$ cells/ $\mu \mathrm{l} ; 95 \% \mathrm{CI}-101$, 137) nor MVM (46 cells/ $\mu 1 ; 95 \%$ CI $-72,165)$ on CD4 counts (interaction, $P=0 \cdot 22$ ), and no effect of any combination of the two interventions (Table 3 ).

\section{Weight gain}

At the 7-month follow-up examination, $389(80.0 \%)$ of the 499 patients were weighed. The mean weight gain was $6.88 \mathrm{~kg}(95 \%$ CI $6.36,7.41)$. However, an interaction was found between $\mathrm{Zn}$ and MVM supplementation $(P=0.03)$. The effect of each combination of Zn and MVM was therefore compared to placebo, based on the use of dummy variables in multiple linear regression analysis (Table 4). As seen, those receiving both $\mathrm{Zn}$ and MVM supplementation had a $2.37 \mathrm{~kg}$ (95\% CI $0.91,3.83 ; P=0.002$ ) greater weight gain than those receiving placebo and placebo, whereas neither $\mathrm{Zn}$ nor MVM alone had any effect. After adjustment for sex, age, HIV status and heavy culture intensity, the estimated effects of $\mathrm{Zn}$ and MVM supplementation combined was $2.63 \mathrm{~kg}$ (95\% CI 1.18, 4.09; $P<0.001$ ), whereas there were no effects of $\mathrm{Zn}$ or MVM alone.

\section{Survival status}

As seen in the trial profile (Fig. 1), survival status could not be ascertained at any time-point after commencement of treatment and supplementation for ten $(2.0 \%)$ of the 499 study participants. Forty-nine $(9.8 \%)$ were lost to follow-up before $210 \mathrm{~d}$ post-treatment, and $77(15.4 \%)$ before $244 \mathrm{~d}$ post-treatment. The median observation time among those lost to full follow-up was $197 \mathrm{~d}$ (interquartile range 121-221d). Hence, $422(84.6 \%)$ of the 499 study participants were known either to have died within $244 \mathrm{~d}$ post-treatment or to be alive after that. There were no differences between the 422 followed

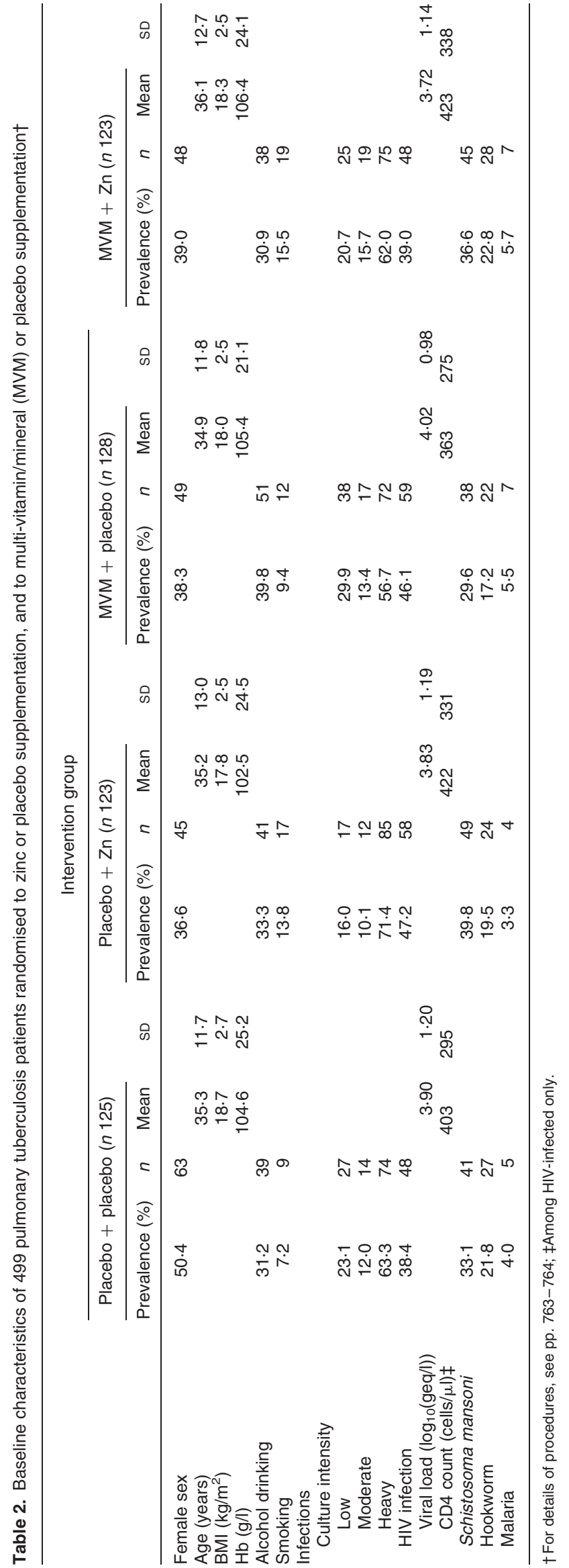


Table 3. Effects of zinc and multi-vitamin/mineral (MVM) supplementation on HIV viral load $\left(\log _{10}(g e q / /)\right)$ and CD4 count (cells/ $\mu$ l) during first 2 months of treatment of pulmonary tuberculosis patients among $213 \mathrm{HIV}$ co-infected patients $\dagger$

\begin{tabular}{|c|c|c|c|c|c|c|c|c|}
\hline & \multicolumn{8}{|c|}{ Intervention group } \\
\hline & \multicolumn{2}{|c|}{ Placebo + placebo $(n$ 48) } & \multicolumn{2}{|c|}{ Placebo + Zn ( $n 58)$} & \multicolumn{2}{|c|}{ MVM + placebo $(n 59)$} & \multicolumn{2}{|c|}{$\mathrm{MVM}+\mathrm{Zn}(n$ 48) } \\
\hline \multicolumn{9}{|l|}{ Viral load } \\
\hline Baseline & 3.90 & $3.53,4.27$ & 3.83 & $3 \cdot 52,4 \cdot 15$ & 4.02 & $3 \cdot 76,4 \cdot 27$ & 3.72 & $3.38,4.05$ \\
\hline 2 months & $4 \cdot 10$ & $3.67,4.54$ & $4 \cdot 28$ & $3 \cdot 86,4 \cdot 71$ & $4 \cdot 14$ & $3.73,4.55$ & 3.85 & $3.46,4.25$ \\
\hline Difference§ & - & & 0.54 & $-0.08,1.15$ & 0.09 & $-0.27,0.55$ & -0.09 & $-0.71,0.54$ \\
\hline \multicolumn{9}{|l|}{ CD4 count } \\
\hline Baseline & 460 & 351,569 & 406 & 327,485 & 365 & 289,442 & 460 & 349,570 \\
\hline 2 months & 403 & 309,569 & 422 & 331,512 & 363 & 281,444 & 423 & 318,529 \\
\hline Change & -104 & $-243,34$ & -9 & $-113,96$ & 19 & 89,127 & -45 & $-175,85$ \\
\hline Difference§ & - & & 96 & $-67,259$ & 124 & $-45,292$ & 60 & $-113,233$ \\
\hline
\end{tabular}

† Data at follow-up available for 184 participants. For details of procedures, see pp. $763-764$

$\ddagger$ Change (2 months - baseline), $t$ test.

$\S$ Difference (each active group - (placebo + placebo)). Linear regression analysis, interaction between interventions, $P=0 \cdot 10$ (viral load) and $P=0 \cdot 18(C D 4$ counts). None were significant.

up and the seventy-seven lost to follow-up with respect to proportion with heavy culture intensity and prevalence of HIV co-infection (Table 5), but the mean viral load among HIV-infected participants was lower among those lost to follow-up. Furthermore, slightly fewer of those lost to follow-up were allocated to $\mathrm{Zn} /$ placebo and more to placebo/MVM. However, the mean observation time among those lost to follow-up was not significantly different between the four groups $(P=0 \cdot 65)$.

Of the $422(84.6 \%)$ patients for which survival status at 8 months was known, fifty-two $(12.3 \%)$ had died: ten $(4.1 \%)$ of the 241 without HIV and forty-two $(23.2 \%)$ of the 181 with HIV co-infection. The median survival time was $106 \mathrm{~d}$ (interquartile range 65-164d).

Of the fifty-two deaths, sixteen occurred in the placebo/placebo, fifteen in the placebo/Zn, thirteen in the MVM/placebo and eight in the MVM/Zn group $(P=0.42)$. Of the forty-two deaths in HIV-infected patients, fourteen were in the placebo/placebo, thirteen in the placebo/Zn, eleven in the $\mathrm{MVM} /$ placebo and four in the MVM/Zn group $(P=0 \cdot 07)$.

Although there was no statistically significant interaction between $\mathrm{Zn}$ and MVM supplementation, the interaction ratio (the RR of MVM supplementation among those given Zn divided by that of those given placebo) was 0.67 for all patients combined, and 0.52 for HIV-infected patients only. The effects of each combination of MVM and Zn supplementation are therefore presented (Table 6). Overall, neither $\mathrm{Zn}$ alone, MVM alone, nor Zn and MVM combined had effects on mortality. For HIV-infected patients only, neither Zn (RR $0.75 ; 95 \%$ CI $0.40,1.42$ ) nor MVM (RR 0.73 ; $95 \%$ CI $0.38,1.43$ ) alone had effects on mortality, whereas MVM and Zn combined considerably reduced mortality (RR 0.29; $95 \%$ CI $0 \cdot 10,0 \cdot 80)$. Similar results were obtained using survival analysis (Fig. 2).

These effect estimates did not change after adjustment for sex, age, alcohol intake or smoking in logistic regression analyses, but adjustment for heavy culture intensity slightly increased the independent effect of $\mathrm{Zn}$ supplementation (RR $0.55 ; 95 \%$ CI $0.31,0.95 ; P=0.03)$ and reduced the independent effect of MVM (RR 0.64; $95 \%$ CI $0.37,1 \cdot 12 ; P=0.12$ ). With similar adjustments, MVM and $\mathrm{Zn}$ together were found to reduce mortality (RR $0.28 ; 95 \%$ CI $0.10,0.77 ; P=0.01$ ), whereas neither Zn (RR 0.66; $95 \%$ CI 0.34, 1.28) nor MVM (RR 0.80; $95 \%$ CI 0.41, 1.55) alone had effects.

Table 4. Effects of zinc and multi-vitamin/mineral (MVM) supplementation on weight gain during treatment of pulmonary tuberculosis among 389 patients weighed at 7 months

\begin{tabular}{|c|c|c|c|c|c|c|c|c|}
\hline & \multicolumn{8}{|c|}{ Intervention group } \\
\hline & \multicolumn{2}{|c|}{ Placebo + placebo ( $n$ 97) } & \multicolumn{2}{|c|}{ Placebo + Zn (n 96) } & \multicolumn{2}{|c|}{ MVM + placebo $(n 101)$} & \multicolumn{2}{|c|}{$\mathrm{MVM}+\mathrm{Zn}(n 95)$} \\
\hline Baseline & $50 \cdot 2$ & $48 \cdot 5,51 \cdot 9$ & $51 \cdot 6$ & $49 \cdot 6,53 \cdot 6$ & $50 \cdot 6$ & $49 \cdot 1,52 \cdot 2$ & $50 \cdot 7$ & $49 \cdot 3,52 \cdot 2$ \\
\hline 7 months & $57 \cdot 9$ & $55 \cdot 8,60 \cdot 0$ & $56 \cdot 2$ & $54 \cdot 5,58 \cdot 0$ & $57 \cdot 2$ & $55 \cdot 6,58 \cdot 7$ & $59 \cdot 4$ & $57 \cdot 8,61 \cdot 0$ \\
\hline Change & $6 \cdot 28$ & $5 \cdot 26,7 \cdot 30$ & $6 \cdot 07$ & $5 \cdot 01,7 \cdot 13$ & $6 \cdot 58$ & $5 \cdot 76,7 \cdot 39$ & 8.65 & $7 \cdot 42,9.88$ \\
\hline
\end{tabular}

Mean value was significantly different from the placebo + placebo group: ${ }^{*} P=0.002$.

†For details of procedures, see pp. 763-764.

$\ddagger$ Change (7 months - baseline), $t$ test.

$\S$ Difference (each active group - (placebo + placebo)). Linear regression analysis, interaction between interventions, $P=0.03$. 
Table 5. Baseline characteristics of the 422 patients followed up for survival status at 8 months compared to 77 not followed up $\dagger$

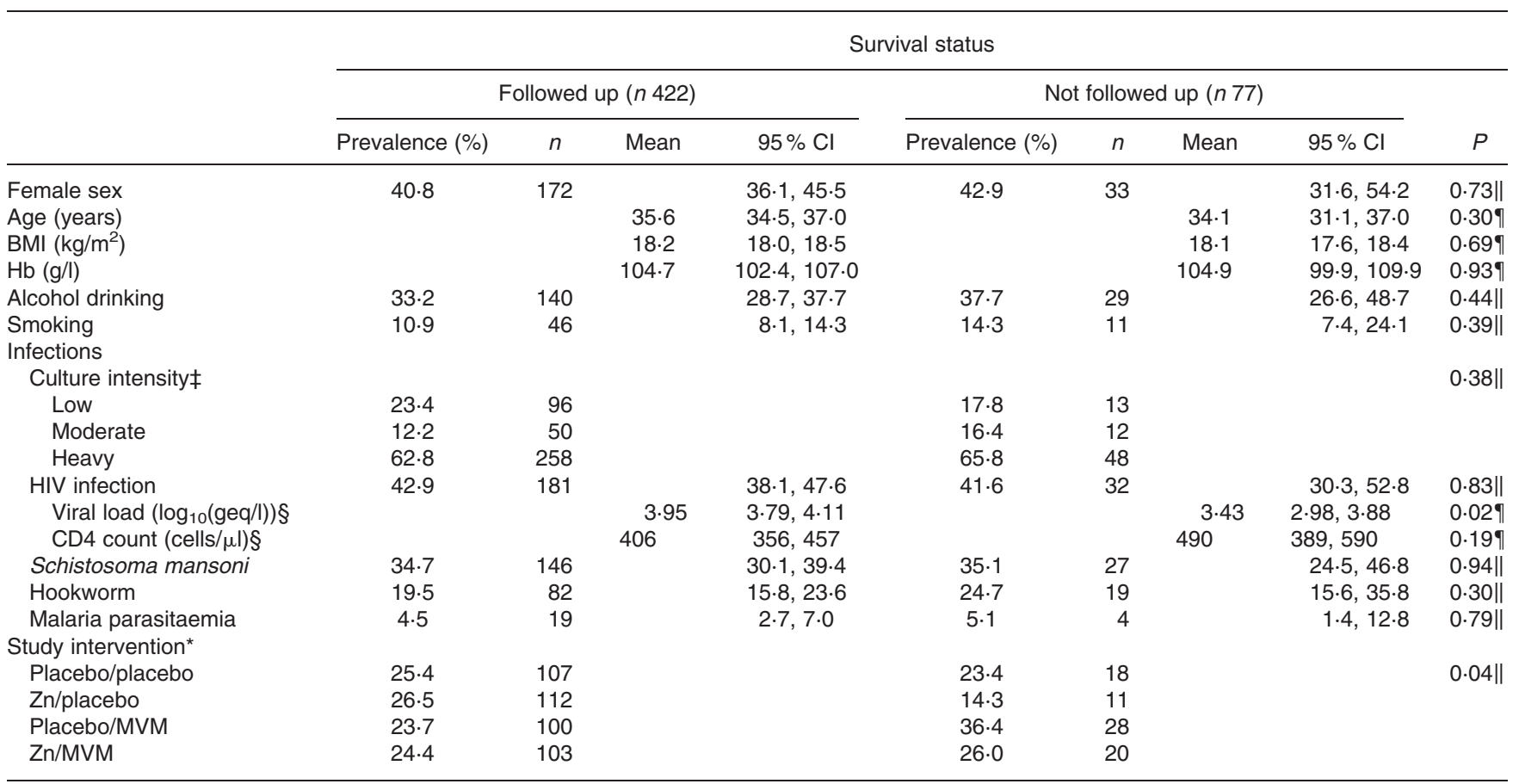

MVM, multi-vitamin/mineral.

*Distribution between intervention groups was significantly different between those followed up and those not followed up. †For details of procedures, see pp. 763-764.

$\ddagger$ Twenty-two were only microscopy-positive: seven were culture-negative, and culture data were missing for fifteen.

$\S$ Among HIV-infected only.

$\| \chi^{2}$ test.

I $t$ test.

\section{Discussion}

The deteriorating TB treatment outcome in the wake of the HIV pandemic is a major concern (UNAIDS, 2004; World Health Organization, 2004), and new adjunctive interventions are needed (Harries et al. 2001). TB patients have considerable weight loss and vitamin and mineral deficiencies at the time of diagnosis (Kennedy et al. 1996; Karyadi et al. 2000; Mugusi e.t al. 2003). Despite appropriate TB treatment, it is unlikely that a patient will be able to regain normal body

Table 6. The effects of different combinations of zinc and multi-vitamin/mineral (MVM) supplementation on cumulative mortality by 8 months among 422 pulmonary tuberculosis patients $\dagger$

\begin{tabular}{|c|c|c|c|c|}
\hline & \multicolumn{4}{|c|}{ Intervention group } \\
\hline & Placebo + placebo & Placebo + Zn & MVM + placebo & $\mathrm{MVM}+\mathrm{Zn}$ \\
\hline All $(n$ 422) & $n 107$ & $n 112$ & $n 100$ & $n 103$ \\
\hline No. of deaths ( $n 52)$ & 16 & 15 & 13 & 8 \\
\hline Mortality (\%) & $15 \cdot 0$ & $13 \cdot 4$ & $13 \cdot 0$ & $7 \cdot 8$ \\
\hline RR $(95 \%$ Cl) $\ddagger$ & - & $0.90(0.47,1.72)$ & $0.87(0.44,1.71)$ & $0.52(0.23,1.16)$ \\
\hline HIV-negative ( $n$ 241) & $n 65$ & $n 60$ & $n 55$ & $n 61$ \\
\hline No. of deaths $(n 10)$ & 2 & 2 & 2 & 4 \\
\hline Mortality (\%) & $3 \cdot 2$ & $3 \cdot 3$ & 3.6 & $6 \cdot 6$ \\
\hline $\mathrm{RR}(95 \% \mathrm{Cl}) \S$ & - & $1.08(0.16,7.45)$ & $1 \cdot 18(0 \cdot 17,8 \cdot 12)$ & $2 \cdot 13(0.40,11 \cdot 21)$ \\
\hline HIV-positive (n 181) & $n 42$ & $n 52$ & $n 45$ & n 42 \\
\hline No. of deaths ( $n$ 42) & 14 & 13 & 11 & 4 \\
\hline Mortality (\%) & $33 \cdot 3$ & $25 \cdot 0$ & $24 \cdot 4$ & 9.5 \\
\hline $\mathrm{RR}(95 \% \mathrm{Cl}) \|$ & - & $0.75(0.40,1.42)$ & $0.73(0.38,1.43)$ & $0.29 *(0.10,0.80)$ \\
\hline
\end{tabular}

RR, relative risk.

Significantly different from the placebo + placebo group: ${ }^{*} P=0.016$.

†For details of procedures, see pp. 763-764.

¥Test for interactions: MVM $\times \mathrm{Zn}, P=0.75 ; \mathrm{MVM} \times \mathrm{HIV}, P=0.02 ; \mathrm{Zn} \times \mathrm{HIV}, P=0.03 ; \mathrm{MVM} \times \mathrm{Zn} \times \mathrm{HIV}, P=0.45$

$\S$ Test for interactions: $\mathrm{MVM} \times \mathrm{Zn}, P=0.66$

\| Test for interactions: MVM $\times \mathrm{Zn}, P=0.30$. 


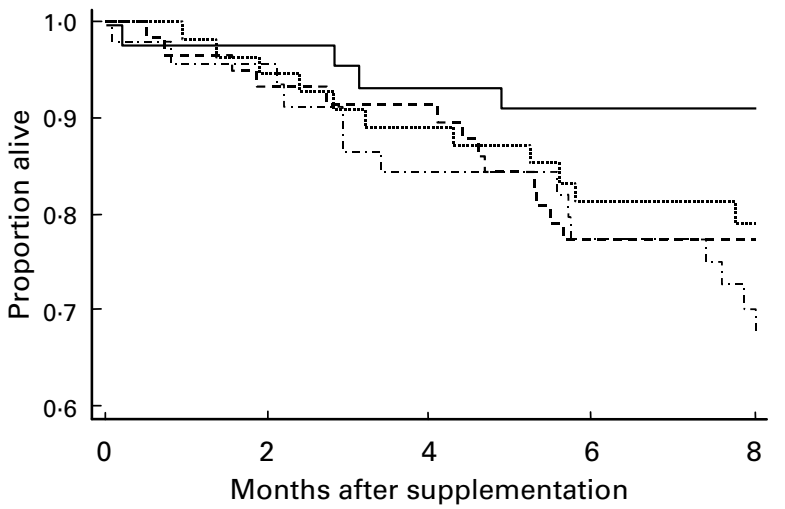

Fig. 2. Kaplan-Meier survival plot by intervention group in 213 HIV coinfected pulmonary tuberculosis patients. - , multi-vitamin/mineral (MVM) + zinc; ....., MVM + placebo; - - - zinc + placebo; ---, placebo + placebo. For details of procedures, see pp. 763-764.

weight and vitamin and mineral status on a typical diet based on cereals, tubers or legumes, which has low content and bioavailability of vitamins and minerals (Ramakrishnan \& Huffman, 2001). The vitamin and mineral deficiencies may impair full restoration of lean body mass and immune functions, and survival.

We therefore assessed the effect of adjunctive $\mathrm{Zn}$ and MVM supplementation given throughout the 8 months of TB treatment. As previously reported, we found no beneficial effect on the primary outcome, sputum conversion which was assessed over the 2-month intensive phase (Range et al. 2005). However, effects on weight gain up to 7-month follow-up, and all-cause mortality during the full 8 months of treatment and supplementation were also assessed.

There were no overall reductions in mortality with $\mathrm{Zn}$ and MVM supplementation. With only ten deaths, the effect of the study interventions could not be assessed among TB patients not infected with HIV. However, among HIV coinfected patients, both $\mathrm{Zn}$ and MVM supplementation were associated with roughly a halving of the risk of death, although not statistically significant, but those receiving the combined supplement had a significant $70 \%$ reduction in risk of death. Due to the deviating interaction ratios it may be considered justified to report the effect of the combined interventions despite the lack of statistical interaction between interventions, since power to detect interactions are often lacking in factorial trials (McAlister et al. 2003).

Survival status could only be ascertained for $85 \%$ of the study participants for the full 8-month treatment period. Nevertheless, the median observation time for those lost to follow-up was above 6 months, and they were similar to those followed up with respect to age, sex, body composition, and the proportion with heavy culture intensity and HIV coinfection, although the mean HIV viral load among infected participants was lower. Among those lost to follow-up, however, a greater proportion was allocated to MVM and a lower proportion to $\mathrm{Zn}$. If the mortality rate among those lost to follow-up was the same irrespective of treatment allocation, then the effect of MVM would be overestimated and the effect of $\mathrm{Zn}$ supplementation underestimated. The proportion receiving both $\mathrm{Zn}$ and $\mathrm{MVM}$ was not different among those lost to follow-up compared to those followed up, so the risk of selection bias seems to be small, and not able to explain the findings. Both patient and investigator were blinded as to the treatment allocation, and data analysis was done prior to breaking the code, based on intention-totreat. Compliance was not systematically assessed, but the supplement was given together with the TB drugs, i.e. under direct observation during the first 2 months and by self-administration during the last 6 months.

This is the first reported randomised trial on the effects of MVM supplementation on mortality among TB patients. However, the effects seen among HIV-infected individuals are in accordance with recent data from randomised trials among HIV-infected individuals in Thailand and Tanzania. The trial from Thailand showed that a daily MVM supplement may reduce mortality among HIV-infected individuals with low CD4 cell counts. The supplement containing twenty-one vitamins and minerals in multiples of RDA was given daily to 481 HIV-infected individuals with CD4 counts between 50 and 550 ( $10^{6}$ cells/1; Jiamton et al. 2003). After 48 weeks, seventynine $(16 \%)$ were lost to follow-up and twenty-three $(5 \%)$ had died. The death rate was lower in those allocated to MVM (mortality hazard ratio $0.53 ; 95 \%$ CI $0 \cdot 22,1 \cdot 25)$, but only significantly so among those with CD4 counts below $100(0 \cdot 26 ; 95 \%$ CI $0 \cdot 07,0.97)$. There was no effect of the intervention on viral load. Similarly, a recent randomised, two-by-two factorial trial among 1078 pregnant HIV-infected women from Dar es Salaam, Tanzania, assessed the effect of a daily vitamin A and multivitamin supplement given throughout pregnancy and for several years after. While vitamin A supplementation increased mother-to-child HIV transmission, multivitamin supplementation increased maternal CD4 cell counts (Fawzi et al. 1999), reduced mother-to-child HIV transmission in subgroups (Fawzi et al. 2002), and also reduced viral load and risk of AIDS and AIDS-related deaths among the mothers themselves (Fawzi et al. 2004).

The supplement we used was similar to the supplement given to pregnant HIV-infected women in Dar es Salaam (Fawzi et al. 1999), but with more vitamin E and less vitamin $\mathrm{C}$, but with addition of vitamin $\mathrm{D}$ and preformed vitamin A, as well as selenium and copper. We were particularly interested in the mineral $\mathrm{Zn}$, since it is known to be essential to the immune system (Shankar \& Prasad, 1998) and host defence to respiratory tract and other infectious diseases (Bhutta et al. 1999, 2000). Therefore, and due to the concern that $\mathrm{Zn}$ may increase progression of HIV in US patients (Tang et al. 1993, 1996), we assessed the effect of $\mathrm{Zn}$ separately, using a two-by-two factorial design. The dose of $\mathrm{Zn}(45 \mathrm{mg}$ ) was considered appropriate, given the high anti-nutrient content of a typical diet in developing countries, and the high requirements during synthesis of lean body mass.

As in the trial in Bangkok, but in contrast to that in Dar es Salaam, the effect of our intervention was apparently not mediated by reduced viral replication, although viral load was only measured over the first 2 months. In contrast, the effect on survival was accompanied by considerable effects on weight gain. Interestingly, neither Zn nor MVM alone had any effects on weight gain, whereas the combined intervention increased weight gain by almost $2.5 \mathrm{~kg}$. This is plausible, since several nutrients are required for the synthesis of new tissues (Golden, 1992). 
Vitamin and mineral supplementation may considerably increase survival during treatment of sputum-positive TB patients co-infected with HIV. Nevertheless, the finding should be confirmed, because the effect is only seen on secondary outcomes in a subgroup of patients, and because compliance data are not available. Also, the effects may differ between different settings, due to interactions with other nutrients and co-infections. The effect should also be assessed among sputum-positive TB patients without HIV co-infection, and among sputum-negative pulmonary TB patients.

The cost is around US $\$ 8$ for the full supply of supplements, which can easily be delivered to the patients together with TB drugs. If proven beneficial, then MVM supplementation could be a feasible and cost-effective adjunctive intervention during TB treatment.

\section{Acknowledgements}

The study was funded by the Danish International Development Assistance through the Council for Development Research and DBL Institute for Health Research and Development.The authors would like to thank all staff from health facilities, laboratories, and the National Institute for Medical Research, Mwanza Centre for their valuable contribution to the study, and the patients for participating. N. R., P. M., A. B. A. and H. F. developed the protocol. N. R. was responsible for the conduct of the trial and for data management, under supervision of J. C., P. M., A. B. A. and H. F.; H. K. was responsible for the viral load determinations. H. F. suggested the study, analysed and interpreted the data and wrote the first version of the manuscript. All authors contributed to the final version of the manuscript. There were no conflicts of interests. The funding source had no influence on study design; collection, analysis and interpretation of data; in the writing of the report; and in the decision to submit the paper for publication.

\section{References}

Beisel WR (2001) Nutritionally acquired immune deficiency syndromes. In Micronutrients and HIV Infection, pp. 23-42 [H Friis, editor]. Boca Raton, FL: CRC Press.

Bhutta ZA, Bird SM, Black RE, et al. (2000) Therapeutic effects of oral zinc in acute and persistent diarrhea in children in developing countries: pooled analysis of randomized controlled trials. Am J Clin Nutr 72, 1516-1522.

Bhutta ZA, Black RE, Brown KH, et al. (1999) Prevention of diarrhea and pneumonia by zinc supplementation in children in developing countries: pooled analysis of randomized controlled trials. Zinc Investigators' Collaborative Group. J Pediatr 135, 689-697.

Cegielski JP \& McMurray DN (2004) The relationship between malnutrition and tuberculosis: evidence from studies in humans and experimental animals. Int J Tuberc Lung Dis 8, 286-298.

Fawzi WW, Mbise RL, Hertzmark E, Fataki MR, Herrera MG, Ndossi G \& Spiegelman D (1999) A randomized trial of vitamin A supplements in relation to mortality among human immunodeficiency virus-infected and uninfected children in Tanzania. Pediatr Infect Dis J 18, 127-133.

Fawzi WW, Msamanga GI, Hunter D, Renjifo B, Antelman G, Bang H, Manji K, Kapiga S, Mwakagile D, Essex M \& Spiegelman D (2002) Randomized trial of vitamin supplements in relation to transmission of HIV-1 through breastfeeding and early child mortality. AIDS 16, 1935-1944.

Fawzi WW, Msamanga GI, Spiegelman D, et al. (2004) A randomized trial of multivitamin supplements and HIV disease progression and mortality. $N$ Engl J Med 351, 23-32.

Food and Nutrition Board (2000) Vitamin C. Dietary Reference Intakes for Vitamin C, Vitamin E, Selenium and Carotenoids. Washington, DC: National Academy Press.

Frieden TR, Sterling TR, Munsiff SS, Watt CJ \& Dye C (2003) Tuberculosis. Lancet 362, 887-899.

Friis H (2005) Micronutrients and HIV Infection: A Review of Current Evidence. www.who.int/nutrition

Friis H \& Sandström B (2001) Zinc and HIV infection. In Micronutrients and HIV Infection, pp. 159-181 [H Friis, editor]. Boca Raton, FL: CRC Press.

Githui W, Kitui F, Juma ES, Obwana DO, Mwai J \& Kwamanga D (1993) A comparative study on the reliability of the fluorescence microscopy and Ziehl-Neelsen method in the diagnosis of pulmonary tuberculosis. East Afr Med J 70, 263-266.

Golden MHN (1992) Nutritional deficiency as a cause of growth failure. In Human Growth: Basic and Clinical Aspects, pp. 175-182 [M Hernández and J Argente, editors]. Amsterdam: Elsevier Science Publishers B.V.

Gomo E, Vennervald BJ, Ndhlovu P, Kaestel P, Nyazema N \& Friis H (2004) Predictors and reference values of CD4 and CD8 T lymphocyte counts in pregnancy: a cross sectional study among HIV negative women in Zimbabwe. Cent Afr J Med 50, 10-19.

Harries AD, Hargreaves NJ, Kemp J, Jindani A, Enarson DA, Maher D \& Salaniponi FM (2001) Deaths from tuberculosis in subSaharan African countries with a high prevalence of HIV-1. Lancet 357, 1519-1523.

Jiamton S, Pepin J, Suttent R, Filteau S, Mahakkanukrauh B, Hanshaoworakul W, Chaisilwattana P, Suthipinittharm P, Shetty P \& Jaffar S (2003) A randomized trial of the impact of multiple micronutrient supplementation on mortality among HIV-infected individuals living in Bangkok. AIDS 17, 2461-2469.

Karyadi E, Schultink W, Nelwan RH, Gross R, Amin Z, Dolmans WM, van der Meer JW, Hautvast JG \& West CE (2000) Poor micronutrient status of active pulmonary tuberculosis patients in Indonesia. J Nutr 130, 2953-2958.

Kennedy N, Ramsay A, Uiso L, Gutmann J, Ngowi FI \& Gillespie SH (1996) Nutritional status and weight gain in patients with pulmonary tuberculosis in Tanzania. Trans $R$ Soc Trop Med Hyg 90, $162-166$.

Krarup HB, Drewes AM \& Madsen PH (1998) A quantitative HCVPCR test for routine diagnostics. Scand J Clin Lab Invest 58, 415-422.

McAlister FA, Straus SE, Sackett DL \& Altman DG (2003) Analysis and reporting of factorial trials: a systematic review. JAMA 289, $2545-2553$.

Ministry of Health (1991) Manual of the National Tuberculosis and Leprosy Programme (NTLP). Geneva: Ministry of Health.

Mugusi FM, Rusizoka O, Habib N \& Fawzi W (2003) Vitamin A status of patients presenting with pulmonary tuberculosis and asymptomatic HIV-infected individuals. Dar es Salaam, Tanzania. Int J Tuberc Lung Dis 7, 804-807.

Paton NI, Chua YK, Earnest A \& Chee CBE (2004) Randomized controlled trial of nutritional supplementation in patients with newly diagnosed tuberculosis and wasting. Am J Clin Nutr 80, 460-465.

Ramakrishnan U \& Huffman SL (2001) Multiple micronutrient malnutrition. In Nutrition and Health in Developing Countries, pp. 365-391 [RD Semba and MW Bloem, editors]. Totowa, NJ: Humana Press.

Range N, Andersen AB, Magnussen P, Mugomela A \& Friis H (2005) The effect of micronutrient supplementation on treatment outcome in patients with pulmonary tuberculosis: a randomized 
controlled trial in Mwanza. Tanzania. Trop Med Int Health 10, 826-832.

Shankar AH \& Prasad AS (1998) Zinc and immune function: the biological basis of altered resistance to infection. Am J Clin Nutr 68, 447S-463S.

Tang AM, Graham NM, Kirby AJ, McCall LD, Willett WC \& Saah AJ (1993) Dietary micronutrient intake and risk of progression to acquired immunodeficiency syndrome (AIDS) in human immunodeficiency virus type 1 (HIV-1)-infected homosexual men. Am J Epidemiol 138, 937-951.

Tang AM, Graham NM \& Saah AJ (1996) Effects of micronutrient intake on survival in human immunodeficiency virus type 1 infection. Am J Epidemiol 143, 1244-1256.

Thurnham DI (2005) An overview of interactions between micronutrients and of micronutrients with drugs, genes and immune mechanisms. Nutr Res Rev 17, 211-240.
UNAIDS (2004) Report on the Global AIDS Epidemic: 4th Global Report. Geneva: UNAIDS.

Wilkinson RJ, Llewelyn M, Toossi Z, Patel P, Pasvol G, Lalvani A, Wright D, Latif M \& Davidson RN (2000) Influence of vitamin D deficiency and vitamin $\mathrm{D}$ receptor polymorphisms on tuberculosis among Gujarati Asians in west London: a case-control study. Lancet 355, 618-621.

World Health Organization (1991) Basic Laboratory Methods in Medical Parasitology. Geneva: WHO.

World Health Organization (1995) Model Prescription Information: Drugs Used in Parasitic Diseases. Geneva: WHO.

World Health Organization (2003) Treatment of Tuberculosis: Guidelines for National Programmes. Geneva: WHO.

World Health Organization (2004) WHO Report 2003. Global Tuberculosis Control: Surveillance, Financing and Planning. Geneva: WHO. 